\title{
PERFIL SICIOECONÔMICO DE GESTANTES CADASTRADAS EM UMA UNIDADE DE SAÚDE DO MUNICÍPIO DE CRICIÚMA
}

\author{
SOCIOECONOMIC PROFILE OF PREGNANT WOMEN REGISTERED IN A \\ HEALTH UNIT THE CITY OF CRICIÚMA
}

\author{
Carla Abbatti Furlanetto ${ }^{1}$ \\ Jayne Fernanda Silveira ${ }^{1}$ \\ Luciane Bisognin Ceretta ${ }^{1,2}$ \\ Lisiane Tuon ${ }^{1,2}$ \\ Priscyla Waleska Simões ${ }^{1,2}$ \\ Rita Suselaine Vieira Ribeiro ${ }^{1,3}$
}

\begin{abstract}
RESUMO
A anemia por deficiência de ferro é definida como a mais prevalente deficiência nutricional do mundo, que acomete todos os países em diferentes graus e tem sua prevalência em regiões de maior vulnerabilidade social. Esse estudo tem como objetivo principal verificar a efetividade do Programa Nacional de Suplementação de Ferro para Gestantes em uma Unidade Básica de Saúde de um município do sul do estado de Santa Catarina. É um estudo quantitativo, transversal e descritivo. A amostra do estudo foi constituída de 40 gestantes em idade de 13 a 37 anos, residentes no bairro São Sebastião. Foram coletadas informações de todos os indivíduos caracterizados na população em estudo, por meio de uma amostra censitária. Das 40 gestantes entrevistadas, perante a escolaridade de cada uma das entrevistadas, $11(26,2 \%)$ tinham ensino fundamental incompleto e outras $11(26,2 \%)$ também possuíam o ensino médio completo. Avaliando a raça, $25(62,5 \%)$ gestantes eram brancas, 8 $(20,0 \%)$ pardas e $7(17,5 \%)$ classificaram-se negras. Entre as gestantes interrogadas predominaram brancas, casadas, moradoras da zona urbana, com baixa escolaridade, que faziam pré-natal regularmente na unidade de saúde e em uso de sulfato ferroso. As gestantes apresentaram idade média de 25 anos e renda per capta de $\mathrm{R} \$ 1.200,00$. Além do fornecimento do ácido fólico e sulfato ferroso, são importantes ações educativas sobre o conhecimento do programa, alimentos fonte de ferro e a importância da suplementação de sulfato ferroso e ácido fólico durante a gestação.
\end{abstract}

Palavras-chave: Anemia Ferropriva; Ácido Fólico; Programa Nacional de suplementação de ferro.

\footnotetext{
${ }^{1}$ Programa de Residência multiprofissional em Atenção Básica/Saúde Coletiva. Universidade do Extremo Sul Catarinense (UNESC), Criciúma, SC, Brasil.

${ }^{2}$ Programa de Pós-Graduação em Saúde Coletiva (PPGSCol). Universidade do Extremo Sul Catarinense (UNESC), Criciúma, SC, Brasil.

${ }^{3}$ Autora para correspondência: Programa de Residência Multiprofissional em Atenção Básica/Saúde da Família. Av. Universitária, 1105 - Criciúma - SC - Bairro Universitário. CEP: 88806-000.E-mail: rsv@ unesc.net.
} 


\begin{abstract}
Anemia due to iron deficiency is defined as the most prevalent nutritional deficiency in the world affecting all countries in varying degrees and has its prevalence in regions of greatest social vulnerability. Its main objective is to verify the effectiveness of the National Program of Iron Supplementation for Pregnant Women in a Basic Health Unit in a city in the southern state of Santa Catarina. A quantitative, descriptive study. The study was conducted at the San Sebastian Neighborhood, Basic Unit located in the town of Crickhowell (Santa Catarina). The study sample consisted of 40 pregnant women aged 13-37 years living in the San Sebastian neighborhood. Information of all individuals featured in the study population through a census sample were collected. Evaluating race 25 (62.5\%) women were white, $8(20.0 \%)$ mixed and $7(17.5 \%)$ classified black. All were performing prenatal (100\%) and only 28 (70.0\%) were using ferrous sulfate supplement provided by the ESF. Among the interviewed pregnant women predominated white, married, living in the urban area, with low education, who were regular prenatal care at the health facility and use of ferrous sulfate. The women had a mean age of 25 years and per capita income of $\mathrm{R} \$ 1,200.00$. Besides the supply of folic acid and ferrous sulphate, it is important actions on educational program awareness, iron-rich foods and the importance of supplementation of ferrous sulfate and folic acid during pregnancy.
\end{abstract}

Keywords: Iron Deficiency Anemia; Folic Acid; National Program for iron supplementation.

\title{
INTRODUÇÃO
}

A anemia por deficiência de ferro é definida como a mais prevalente deficiência nutricional do mundo, que acomete todos os países em diferentes graus e tem sua prevalência em regiões de maior vulnerabilidade social (KALAIVANI, 2009). É resultante da interação de vários fatores etiológicos que induzem um desequilíbrio entre as necessidades do organismo e a quantidade absorvida de ferro (KALAIVANI, 2009)

O consumo dietético insuficiente de alimentos ricos em ferro é um dos principais fatores associados à deficiência de ferro e à anemia ferropriva, especialmente em mulheres de países em desenvolvimento, as quais muitas vezes apresentam consumo insuficiente de ferro, de modo que a maioria não tem reservas suficientes desse mineral para suprir a demanda elevada que ocorre durante a gestação. Desse modo, conhecer a ingestão dietética de ferro e os fatores a ela associados em gestantes é primordial para identificar aquelas em risco de consumo inadequado, especialmente em populações de baixo ou médio desenvolvimento socioeconômico (KALAIVANI, 2009).

A anemia por deficiência de ferro é a carência nutricional de maior magnitude no mundo, sendo considerada uma carência em expansão em todos os segmentos sociais, atingindo principalmente crianças menores de dois anos e gestantes. Embora ainda não haja um levantamento nacional, estudos apontam que aproximadamente metade dos pré-escolares brasileiros sejam anêmicos (cerca de 4,8 milhões de crianças) com a prevalência chegando a 
67,6\% nas idades entre 6 e 24 meses. No caso de gestantes, estima-se uma média nacional de prevalência de anemia em torno de 30\% (WORLD HEALTH ORGANIZATION, 2001).

A anemia ferropriva está entre as causas mais comuns de anemia na gestação, e diretamente relacionada aos mecanismos de expansão do volume sanguíneo e plasmático que aumentam em torno de $50 \%$ durante a gestação. Além disso, observa-se um aumento das necessidades de ferro para o desenvolvimento do feto, da placenta e cordão umbilical, e para as perdas sanguíneas por ocasião do parto e puerpério (OLIVEIRA et al., 2002).

O aumento da prevalência da anemia ferropriva em crianças pode ser decorrente das mudanças nos hábitos alimentares, que acompanham a transição nutricional no país. No Brasil, a tendência do aumento da anemia em pré-escolares foi evidenciada por dois estudos nos quais a prevalência da doença passou de 35,6\% na década de 1980, para 46,9\% na década de 1990, no município de São Paulo, e de 19,3\% para 36,4\%, na Paraíba (NASCIMENTO et al., 2001).

Devido à elevada prevalência o combate à anemia ferropriva é uma das prioridades para os profissionais responsáveis pelo planejamento de Programas de Nutrição em Saúde Pública, encontrando respaldo político no compromisso social assumido pelo Brasil de reduzir a anemia por carência de ferro. O Ministério da Saúde tornou obrigatória a fortificação das farinhas de milho e trigo com ferro e ácido fólico, por serem alimentos de fácil acesso a população e não terem alterações de suas características organolépticas no processo de fortificação, além de ser economicamente viável ao país. A partir de 1998 foi implantado o Programa Nacional de Suplementação Medicamentosa de ferro aos grupos de risco (crianças de 6 a 18 meses, gestantes e mulheres no pós-parto)(IBGE, 2002).

Nesse contexto, essa pesquisa busca verificar a efetividade do Programa Nacional de Suplementação de Ferro para Gestantes e o perfil socioeconômico das gestantes adstritas em uma Unidade de Saúde de um município do Sul do estado de Santa Catarina.

\section{METODOLOGIA}

Estudo quantitativo, transversal e descritivo, aprovado pelo Comitê de Ética em Pesquisa do local onde foi realizada a pesquisa com parecer $n^{\circ}$ 400.296/2013.

A população foi composta por 40 gestantes adstritas no Bairro São Sebastião, localizado no município de Criciúma (Santa Catarina). Foram excluídas as gestantes que não estavam sendo acompanhadas na Estratégia Saúde da Família São Sebastião, que se opuseram a responder o questionário e não aceitaram assinar o termo de consentimento livre e esclarecido. 
Foi elaborado um questionário fechado sobre suplementação de ferro e ácido fólico elaborado pelos autores dessa pesquisa, assim, as gestantes foram questionadas na unidade de saúde antes dos atendimentos clínicos individuais.

Foram utilizadas no questionário as variáveis Escolaridade, Raça, Estado Civil, Zona de Residência, Realização do Pré-Natal, Quem indicou, Considera a suplementação importante, Usou Sulfato ferroso/Ácido fólico nos últimos 6 meses, Nas outras gestações usou ferro e Ácido fólico.

Para a análise estatística foi organizado um banco de dados em planilhas do software Microsoft Excel versão 2010, onde foram construídos gráficos e tabelas para uma melhor organização e apresentação dos dados. Também foram calculadas algumas medidas descritivas como média e desvio padrão para as variáveis quantitativas, e frequência absoluta e relativa para as qualitativas.

Em seguida, o banco de dados foi exportado para o software Statistical Package for the Social Sciencies (SPSS) versão 20.0, onde foi realizada a análise estatística.

Foi avaliada a normalidade dos dados pelo Teste de Shapiro-Wilk, e para as variáveis que não apresentaram distribuição gaussiana, foi calculada a mediana e o intervalo interquartil (quantidade de gestações e renda).

\section{RESULTADOS}

As gestantes entrevistadas apresentaram idade média de 25 anos $( \pm 5,93)$, considerando que a mais nova tinha 13 anos e a mais velha tinha 37 anos; renda per capita de $\mathrm{R} \$ 1.200,00$ $( \pm \mathrm{R} \$ 840,00)$ e a mediana das gestações foi de $1,00( \pm 1)$.

Ao detalhar os resultados das entrevistas apresentadas na Tabela 1, pode-se perceber que nenhuma das gestantes se declararam analfabetas, mais da metade possuem ensino fundamental completo e incompleto (52,5\%), 17 (42,5\%) ensino médio completo e incompleto, $1(2,5 \%)$ ensino superior incompleto e apenas $1(2,5 \%)$ ensino superior completo.

Avaliando a raça, $25(62,5 \%)$ gestantes se declararam brancas, $8(20,0 \%)$ pardas e 7 $(17,5 \%)$ classificaram-se como negras.

A maioria dos entrevistados, 39 (97,5\%), morava na área urbana do bairro. Das gestantes entrevistadas, $34(85,0 \%)$ eram casadas ou estavam em uma união estável, 5 $(12,5 \%)$ solteiras e apenas $1(2,5 \%)$ em processo de separação.

Todas estavam realizando o pré-natal $(100,0 \%)$ ou dando início ao mesmo, e apenas $28(70,0 \%)$ estavam usando suplemento de sulfato ferroso fornecido pela ESF, pois as outras ainda não tinham passado pelo médico da unidade. 
A maioria das gestantes acha importante a suplementação $(87,5 \%), 12,5 \%$ não responderam ou não sabem informar por que achavam importante a suplementação de ácido fólico/sulfato ferroso durante a gestação.

Aproximadamente $37(92,5 \%)$ das gestantes não usaram ácido fólico ou sulfato ferroso nos 6 meses antecedentes à gestação e apenas 3 (7,5\%) gestantes fizeram uso.

Tabela 1. Características das gestantes adstritas no bairro São Sebastião.

\begin{tabular}{lc}
\hline Variável & $\mathbf{N}(\mathbf{\%})$ \\
& $\mathbf{N}=\mathbf{4 0}$ \\
\hline Escolaridade & \\
Ensino fundamental incompleto & $11(27,5)$ \\
Ensino fundamental completo & $10(25,0)$ \\
Ensino médio incompleto & $6(15,0)$ \\
Ensino médio completo & $11(27,5)$ \\
Ensino superior incompleto & $1(2,5)$ \\
Ensino superior completo & $1(2,5)$ \\
Raça & \\
Branca & $25(62,5)$ \\
Parda & $8(20,0)$ \\
Negra & $7(17,5)$ \\
Zona de Residência & $39(97,5)$ \\
Urbana & $1(2,5)$ \\
Urbana/Rural & \\
Estado Civil & $5(12,5)$ \\
Solteira & $34(85,0)$ \\
Casada & $1(2,5)$ \\
Separada & \\
Realização do Pré-Natal & $40(100,0)$ \\
Sim & \\
Quem indicou? & $28(70,0)$ \\
Médico & $1(2,5)$ \\
Enfermeira & \\
Considera a suplementação importante? & $35(87,5)$ \\
Sim & \\
Usou Sulfato ferroso/Ácido fólico nos & \\
últimos 6 meses? & $25,03(5,93)$ \\
Sim & $1200,00(\mathrm{R} \$ 840,00)$ \\
Não & $1,00(1)$ \\
Nas outras gestações usou ferro? & \\
Sim & $3(7,5)$ \\
Não & $35(87,5)$ \\
E Ácido fólico? & $20(50,0)$ \\
Sim & $3(7,5)$ \\
Não & \\
Média de Idade (DP) & $10(25,0)$ \\
Mediana da renda (II) & \\
Mediana das Gestações (II) & \\
\hline & \\
\hline
\end{tabular}

Quando questionadas se usaram ácido fólico ou sulfato ferroso nas outras gestações, $20(50,0 \%)$ gestantes responderam que o utilizaram e $3(7,5 \%)$ não usaram. Sobre o uso de 
ácido fólico, 10 (25,0\%) gestantes usaram e 7 (17,5\%) não fizeram uso. É importante considerar que algumas gestantes não lembravam se tinham feito uso ou não, e outras estavam na primeira gestação, portanto não tinham antecedentes.

$\mathrm{Na}$ análise da mediana das gestações e a escolaridade das mães, percebe-se que mães com menor grau de instrução possuíam um número maior de filhos conforme ilustra a Tabela 2.

Tabela 2. Relação entre escolaridade e quantia de gestações das gestantes adstritas no bairro São Sebastião.

\begin{tabular}{lccc}
\hline \multicolumn{1}{c}{ Escolaridade } & Mediana das Gestações & $\begin{array}{c}\text { Intervalo } \\
\text { interquartil }\end{array}$ & P \\
\hline Ensino Fundamental incompleto & 3,0 & 1,0 & 0,19 \\
Ensino Fundamental completo & 1,0 & 0,0 & \\
Ensino Médio incompleto & 1,0 & 1,0 & \\
Ensino Médio completo & 2,0 & 1,0 & \\
\hline
\end{tabular}

\section{DISCUSSÃO}

Foram identificadas na Unidade Básica de Saúde cerca de 60 gestantes cadastradas durante o período de agosto a novembro de 2013, sendo incluídas na pesquisa 40 gestantes, pois as demais realizaram o pré-natal em outras Unidade de Saúde ou em outros Serviços Privados de Saúde.

Nas últimas décadas, o tamanho das famílias vem sofrendo reduções sistemáticas, como reflexo da queda da fecundidade. Em 1980, as famílias brasileiras tinham, em média, 4,5 componentes (PACHECO et al., 2009). Em 1992, o tamanho médio passou para 3,7 pessoas e, em 2001, chegou a apenas 3,3 pessoas em média. Em termos regionais, a média mais elevada se encontra nas regiões Norte e Nordeste, ambas com média de 3,7 componentes por família, enquanto no Sul e no Sudeste o tamanho médio é 3,2 pessoas.

No país, o tipo predominante de família ainda é casal com filhos (53,3\% em 2001), mas a participação desse arranjo no total caiu desde 1992 (59,4\%), seguindo uma trajetória de queda já verificada nas décadas de 1980 e 1990. Paralelamente, confirma-se a tendência de crescimento da proporção de famílias compostas por mulheres sem cônjuge e com filhos, passando de 15,1\% em 1992 para 17,1\% em 1999 e atingindo 17,8\% em 2001. A análise regional aponta o Sudeste como a região brasileira com menor proporção do arranjo tradicional $(52,3 \%)$, dando margem ao crescimento proporcional dos tipos alternativos de famílias, como as unipessoais e aquelas compostas por mulheres sem cônjuge e com filhos 
(PACHECO et al., 2009), colaborando com nosso estudo onde 85,0\% das gestantes estão em uma união estável e apenas $12,5 \%$ são solteiras.

Ainda corroborando com nossa casuística, mesmo que a taxa de fecundidade da brasileira de forma geral tenha caído, persistem discrepâncias entre as Grandes Regiões e o nível de escolaridade das mulheres. As mulheres com maior escolaridade (8 anos ou mais) apresentaram em nossa amostra, em média, 1,7 filhos, e as com menos de 4 anos de estudo tinham 3,7 filhos. Essa influência da escolaridade na fecundidade é observada em todas as regiões brasileiras, as maiores médias de filhos foram observadas no Norte $(3,2)$ e no Nordeste $(2,7)$, mas essas duas regiões foram as que apresentaram maior queda neste indicador (16,7\% entre 1992 e 2001), assim como na tabela 2, onde mostra que as média das gestações comparado com a escolaridade das gestantes é de 3 gestações em mulheres com Ensino Fundamental Incompleto(PACHECO et al., 2009).

Em um estudo realizado com puérperas no município de Rio Grande (RS), foram avaliadas 2.557 mulheres, no ano de 2007, dessas, apenas $60 \%$ das entrevistadas receberam suplemento de sulfato ferroso durante o pré-natal, e 70\% eram de cor branca (PAIXÃO et al., 2012).

Quanto a adesão das gestantes ao tratamento com o suplemento de sulfato ferroso, em um estudo realizado em São Leopoldo-RS, das 76 gestantes que estavam no grupo de intervenção, 88,2 \% referiram usar o suplemento, sendo que dessas 67,7\% fizeram uso diário, $24,9 \%$ não o fizeram e 7,4\% usaram apenas metade da dose prescrita, colaborando com nossa casuística (NASCIMENTO et al., 2001).

O ácido fólico é o mais importante fator de risco para os defeitos do tubo neural identificado até hoje. A suplementação periconcepcional e durante o primeiro trimestre de gravidez tem reduzido tanto o risco de ocorrência como o risco de recorrência para os defeitos do tubo neural em cerca de $50 \%$ a $70 \%$. Devido à gravidade dos defeitos do tubo neural e sua morbimortalidade, torna-se importante o aconselhamento genético, a suplementação dietética com ácido fólico e o diagnóstico pré-natal das malformações do tubo neural (BRASIL MINISTÉRIO DA SAÚDE, 2014). Quando questionadas sobre o uso, 25,0\% das gestantes referiram o uso do ácido fólico em gestações anteriores.

A carência materna de ferro durante o período gestacional também pode comprometer o desenvolvimento do cérebro do recém-nascido, levando ao prejuízo no desenvolvimento físico e mental, diminuição da capacidade cognitiva, aprendizagem, concentração, memorização e alteração do estado emocional (ORGANIZAÇÃO MUNDIAL DA SAÚDE, 
2013) quanto ao sulfato ferroso, em nossa população, 50,0\% das gestantes responderam que usaram.

Realizado com 74 adolescentes grávidas entre 13 e 18 anos, que encontravam-se no período de 16 a 20 semanas de gravidez, concluiu-se que o uso de $80 \mathrm{mg}$ e $120 \mathrm{mg}$ de ferro em adolescentes grávidas provoca similar melhora na resposta hemoglobínica, porém, não interfere na reserva do ferro, podendo estar associado ou não ao ácido fólico e ao zinco (NOGUEIRA; PARENTE; COZZOLINO, 2003).

\section{CONCLUSÕES}

Entre as gestantes interrogadas predominaram brancas, casadas, com baixa escolaridade, moradoras da zona urbana, que faziam pré-natal na Unidade Básica de Saúde e em uso de sulfato ferroso orientadas pelo médico.

As políticas públicas são necessárias para informar e divulgar a população sobre as carências nutricionais que podem atingir as gestantes e, por conseguinte, os recém-nascidos. Essas informações devem ir não somente ao grupo materno, mas também a todos os envolvidos durante esse período pré-natal, como os familiares que convivem com as gestantes e as unidades de saúde, que dão esse suporte durante todo este período.

Além do fornecimento do ácido fólico e sulfato ferroso, é importante a realização de ações educativas sobre o conhecimento do programa Suplementação de Ferro, quais os alimentos fonte desse nutriente, qual a importância da suplementação do sulfato ferroso e ácido fólico durante a gestação, das melhorias da adesão na gestação e continuação do tratamento.

Sugerem-se futuras pesquisas para ampliar o assunto, e entender a sua devida importância, como, por exemplo, a atualmente realizada pelo Ministério da Saúde, que busca avaliar a efetividade, aceitação e adesão da fortificação caseira com vitaminas e minerais na prevenção da anemia e deficiência de ferro em crianças menores de um ano segundo modelo de atenção básica, sendo um estudo multicêntrico realizado em quatro cidades brasileiras, totalizando um público amostral de 1.350 crianças (BRASIL, 2014).

A suplementação diária oral desses dois nutrientes age como conjunto da assistência ao pré natal para reduzir o risco de baixo peso no nascimento, anemia materna e deficiência de ferro, sendo sugerido então o seguinte esquema: 30 a 60mg de ferro $+400 \mu \mathrm{g}$ de ácido fólico, sendo suplementados uma vez ao dia durante toda a gestação, evitando, por fim, as consequências nutricionais (ORGANIZAÇÃO MUNDIAL DA SAÚDE, 2013). 


\section{REFERÊNCIAS}

BRASIL. Ministério da Saúde. Portal da Saúde. 2014.

IBGE. Síntese de indicadores sociais. IBGE, 2002.

KALAIVANI, K. Prevalence \& consequences of anaemia in pregnancy. Indian Journal of Medical Research, v. 130, n. 5, p. 627-33, Nov 2009.

NASCIMENTO, N. N. et al. Utilização de diferentes concentrações de ferro por adolescentes grávidas também suplementadas com zinco e ácido fólico. Archivos Latinoamericanos de Nutrición, v. 51, p. 225-229, 2001.

NOGUEIRA, N. D. N.; PARENTE, J. V.; COZZOLINO, S. M. F. Mudanças na concentração plasmática de zinco e ácido fólico em adolescentes grávidas submetidas a diferentes esquemas de suplementação. Cadernos de Saúde Pública, v. 19, p. 155-160, 2003.

OLIVEIRA, R. S. D. et al. Magnitude, distribuição espacial e tendência da anemia em préescolares da Paraíba. Revista de Saúde Pública, v. 36, p. 26-32, 2002.

ORGANIZAÇÃO MUNDIAL DA SAÚDE. Diretriz: Suplementação diária de ferro e ácido fólico em gestantes. Genebra: Organização Mundial da Saúde, 2013.

PACHECO, S. S. et al. Efeito da fortificação alimentar com ácido fólico na prevalência de defeitos do tubo neural. Revista de Saúde Pública, v. 43, p. 565-571, 2009.

PAIXÃO, G. P. D. N. et al. A importância do uso do ácido fólico e sulfato ferroso em mulheres no planejamento familiar e ciclo gravídico-puerperal: revisão integrativa da literatura. Revista de APS, v. 15, n. 2, 2012.

WORLD HEALTH ORGANIZATION. Iron deficiency anaemia: assessment, prevention and control. Geneva: World Health Organization, 2001. 